\title{
Hierarchical Hashing for Image Retrieval
}

\author{
Cheng Yan*, Xiao Bai* Jun Zhou ${ }^{\dagger}$ Yun Liu ${ }^{\ddagger}$ \\ * School of Computer Science and Technology, Beihang University \\ Email: beihangyc,baixiao@buaa.edu.cn \\ ${ }^{\dagger}$ School of Information and Communication Technology, Griffith University \\ Email: jun.zhou@griffith.edu.au \\ ${ }^{\ddagger}$ School of Automation Science and Electrical Engineering, Beihang University \\ Email: liuyun@buaa.edu.cn
}

\begin{abstract}
Hashing has been widely used in large-scale vision problems thanks to its efficiency in both storage and speed. The quality of hashing can be boosted when supervised information is used to learn hash functions. On large-scale hierarchical datasets, hierarchical semantic information reflects the relationship between classes and their children, which however has been ignored by most supervised hashing methods. In this paper, we propose a hierarchical hashing method for image retrieval. This method models and fuses both hierarchical semantic level relationship through taxonomy structure of dataset and feature level relationship of images into an integrated learning objective, then an optimization scheme is developed to solve the learning problem. Experiments are performed on two large-scale datasets: ImageNet ILSVRC 2010 and Animals with Attributes (AWA) dataset. Besides standard evaluation criteria, we also developed hierarchical evaluation criteria for image retrieval and classification tasks. The results show that the proposed method improves the accuracy of supervised hashing in both types of criteria.
\end{abstract}

\section{INTRODUCTION}

Nearest neighbor (NN) search has been widely adopted in image retrieval. The time complexity of the $\mathrm{NN}$ method on a dataset of size $n$ is $O(n)$, which is infeasible for realtime retrieval on large dataset. Approximate nearest neighbor (ANN) search has been proposed to make NN search scalable, and becomes a preferred solution in many computer vision and machine learning applications [1], [2]. The goal of ANN search is to find approximate results rather than exact ones so as to achieve high speed data processing [3], [4], [5], [6]. Amongst various ANN search techniques, hashing is widely studied because of its efficiency in both storage and speed. By generating binary codes for image data, image retrieval on a dataset with millions of samples can be completed in a constant time using only tens of hash bits [7], [8].

Classic hashing methods, such as locality sensitive hashing (LSH) [9], try to guarantee that close samples in the original space having similar binary codes. These methods are dataindependent hash, which do not employ a training set to learn the hash function. Besides LSH, some other data-independent hashing schemes have been proposed [10], [11], [12]. For example, Raginsky and Lazebnik [12] utilized random Fourier features to make the Hamming distance be related to the shiftinvariant kernel between vectors. Such methods usually require long hash bits to achieve good performance, which may result in low recall. To address this problem, many researcher developed data-dependent approaches [13], [14], [15] to generate compact set of hash bits from image datasets. A representative data-dependent hashing scheme is spectral hashing ( $\mathrm{SH})$ [16]. It transforms the problem of finding similarity preserving code for a given dataset to an NP-hard graph partitioning problem that is similar to Laplacian eigenmaps [17]. SH relaxes this problem and solves it by a spectral method [16], [17]. For novel data point, SH uses the Laplace-Beltrami eigenfunctions to obtain binary codes under the hypothesis that the data is uniformly distributed. To address the problem when data do not meet this hypothesis, anchor graph hashing (AGH) [18] has been proposed. AGH uses an anchor graph to obtain a low-rank adjacency matrix which is computationally feasible to approximate the similarity matrix and then processes it in constant time based on the Nyström method [19]. These approaches can be divided into unsupervised [20], [18], [16] and supervised [21], [22], [2] depending on whether they use label information. Most unsupervised hashing methods [20], [16], [18] retrieve neighbors based on metric distance, ignoring the semantic information. Supervised methods take full advantage of the annotation of the dataset and have higher search accuracy than unsupervised hashing methods.

Some supervised methods take simple semantic relationship of data into consideration [23], [22]. Semi-supervised hashing ( $\mathrm{SSH})$ [8] has been introduced to search semantic similar instances when only part of the data are labelled. It minimizes the empirical error over the labeled data, and applies an information theoretic regularizer over both labeled and unlabeled data. Projection learning hashing method [24] has been proposed in a similar form as SSH, containing a semisupervised method and an unsupervised method. Beside SSH, weakly-supervised hashing [25] and kernel-based supervised hashing (KSH) [26] are two other supervised hashing schemes that have kernel based hash functions. Kulis et al. extended LSH functions to a learned metric [27], which can also be considered as a supervised method. In [20], Gong et al. also presented a supervised hashing method CCA-ITQ based on Canonical Correlation Analysis (CCA) and an iterative quantization method. In [28], Shen et al. formulate the hashing framework with classification to learn hash codes expected to be optimal for classification, which also is a supervised hash method.

Though these supervised methods achieve good performance for retrieval, when dealing with multi-class data with 

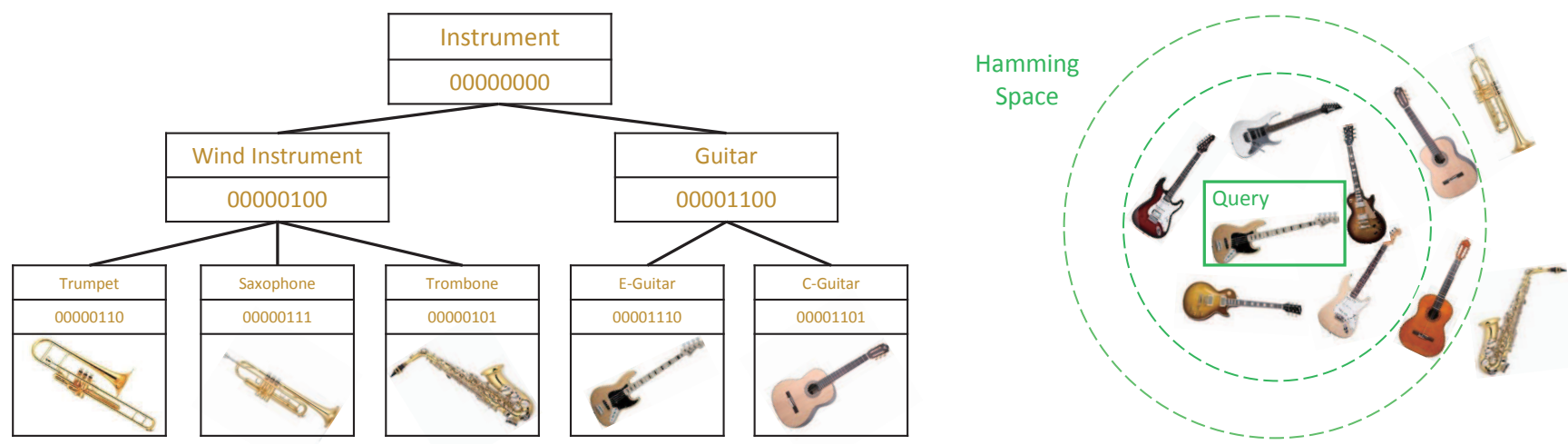

Fig. 1. An example that shows the hierarchical semantic structure of data. The images and structural relationship are extracted from the ImageNet Large Scale Visual Recognition Challenge 2010 (ILSVRC2010). Classic guitar and electric guitar are different objects. However, they belong to the same category of guitar. Their hash codes shall be different but with short Hamming distance. The right part is a sketch map to show the distances between a query and retrieval results in the Hamming space.

complex taxonomy structure, most of them are not effective because they are developed on flat datasets in which the relationship of the categories of data is relatively simple. Over the past few years, there have been growing interests in building large-scale sets with hundreds or thousands of classes with hierarchical structure for computer vision tasks. ImageNet [29], as an example, is organized according to the Wordnet hierarchy. There can be thousands of nodes in the hierarchy, each of which represents a class and each node is depicted by hundreds and thousands of images. Some researchers focus on studying the semantic taxonomy to finish vision tasks [30], [31]. They learn or construct specific structure for datasets to facilitate classification or recognition. For image retrieval, especially for hash retrieval, the hierarchy semantic information shall also be considered.

Suppose Figure I is a dataset and we have an image of E-guitar as a query. If the retrieval result is a group of images of E-guitar, it is absolutely the best result. If it returns the images of Trumpet, Saxophone, or Trombone, which are not Guitar, not to mention E-guitar, we consider the retrieve result as unsatisfactory. If it returns C-guitar, the result is quite good, since the C-guitar looks a lot like E-guitar and they belong to the same category Guitar.

In this paper, we study hierarchical hashing for image retrieval on large-scale dataset. Figure I shows a simple example of the hierarchical dataset which is extracted from a subset of ImageNet. In real world, objects in similar categories may have similar appearance. They are usually from the same semantic category such as Trombone and Trumpet under Wind Instrument in Figure I. Though their names are different, sometimes the names may have a common keyword, for example, C-Guitar and E-Guitar under Guitar. In a retrieval system, it is natural to enforce the samples between C-Guitar and E-Guitar categories to have more common codes than between C-Guitar and Trumpet categories.

Based on this motivation, our work aims to develop a supervised hashing retrieval method that explores hierarchi- cal semantic information on large-scale hierarchical dataset. The structural semantic information is adopted to model the similarity between classes, which is then used to construct a label matrix. The label matrix is then used to learn the hash functions. More than that, we add supervised clustering optimization to the objective functions according to the hierarchical structure, which guarantees that the feature level semantic relationship between classes be maintained in the hash codes. By taking consideration of both semantic level and feature level relationships for hashing learning, dissimilar categories are assigned with large hamming distances in the binary space (like C-Guitar and Trumpet in Figure I), and similar categories with different hash codes but more common bits.

There are three contributions of our work. First, we propose a hierarchical hashing for image retrieval on large-scale datasets by combining both semantic level relationship and feature level relationship into a hashing learning objective. Second, we develop an effective optimization solution for solving the learning objective. Third, we boost retrieval and classification performance with respect to both general evaluation criteria and specific hierarchical criteria for hierarchical datasets.

This paper is organized as follows. Section II introduces related work. Section III describes our hashing framework. The experimental results are presented in Sections IV. The conclusions are drawn in Section $\mathrm{V}$.

\section{RELATED WORK}

ImageNet [29] is a large visual database built for computer vision tasks such as recognition and classification. Some researchers study the semantic taxonomy to complete vision tasks on ImageNet. Verma et al. [30] proposed a method using taxonomy to learn similarity metrics. They showed that the learned metrics lead to improved classification performance. The metrics can provide effective classification amongst categories local to specific subtrees of the taxono- 
my. Zhao et al. [31], on the other hand, focused on using semantic taxonomy to guarantee classification accuracy and reduce dimensionality of parameter space at the same time. Nonetheless these research explored the semantic taxonomy of the hierarchical dataset, there are few works that explore the semantic taxonomy of dataset effectively for fast image retrieval.

Supervised hashing methods, such as semantic hashing (SH) [22], learning to hash with binary reconstructive embeddings (BRE) [32], minimal loss hashing for compact binary codes (MLH) [23], supervised hashing with kernels (KSH) [26], and supervised Discrete Hashing (SDH) [28], try to make the hash codes keep semantic similar or dissimilar relationship between samples. Most of them minimize the discrepancy between the data similarities and the Hamming distances of hash codes. During the training process, labels are provided to model the similarity/dissimilarity of data. For example, KSH [26] uses label 1 to specify similar pairs, label -1 to designate dissimilar pairs, and 0 for the unknown pairs. However, simply dividing the sample relationship into several specific values are not sufficient for characterizing the complex taxonomy structure in very large datasets. Our work takes such taxonomy structure into consideration, in which the hash codes of data pairs are partly based on their distances in the semantic structure.

Large-scale training sets is key to achieving good performance for many vision tasks [33], [3], [5]. Large datasets with taxonomy structure, e.g. ImageNet and ILSVRC [29], have been adopted by many retrieval or classification tasks [34], [30], [31]. Krizhevsky et al. [34] trained a deep convolutional neural network for classification on hierarchical datasets. Liong et al. [35] used CNN for binary code learning. Though having good performance, these methods have to learn a large number of model parameters. Verma et al. [30] proposed a framework based on probabilistic nearest-neighbor classification, aiming at learning similarity metrics using the class taxonomy, with focuses on using metrics for classification. Hashing was used to reduce the dimensionality of parameter space, but only for classification. Deng et al. [36] exploited prior knowledge of a semantic hierarchy for image retrieval. Its hashing retrieval method is close to LSH [9] which has advantage in efficiency but not accuracy. Our work is a hashing based solution, using hierarchy semantic information of dataset to learn projection for image retrieval. Meanwhile it also can be used for classification tasks.

\section{Hierarchical Hashing Method}

The basic idea of hashing is to project data onto a lowdimensional space so that each data is mapped to a binary vector. When a query arrives, the most similar items in the dataset are retrieved by comparing the mapped vectors (hash key) of the query and the dataset. Different from general hashing, our method is based on the hierarchical structure of the dataset. After training, each node in the hierarchy is also assigned with a hash key. This enables hierarchical retrieval.
In our work, we assume that categories are organized in a tree structure like the ImageNet. Only the leaf nodes contain image data. The hash functions are learned using both feature level information of training data and the hierarchical structure information. The learned hash functions can be used to encode both training and query data, from leaf to intermediate nodes, so the goal of our method is to generate the hash function and the binary hash code for every node.

\section{A. Prediction Function}

The purpose of hashing is to derive a group of hash functions accounting for the hash bits generation. Given a training set $\mathbf{X}=\left\{\mathbf{x}_{1}, \mathbf{x}_{2}, \ldots, \mathbf{x}_{n}\right\} \subset \mathbb{R}^{d}$, we use a kernel formulation to cope with linearly inseparable data in the target hash functions. We first define a prediction function

$$
y=\operatorname{sgn}\left(\sum_{i=1}^{m} \kappa\left(\mathbf{x}_{i}, \mathbf{x}\right) w_{i}-b\right)
$$

where $y$ is a single bit hash code for the sample $\mathbf{x} . \mathbf{x}_{1}, \ldots, \mathbf{x}_{m}$ are $m$ samples uniformly and randomly selected from the training set and $m \ll n$ in which $n$ is the size of the training set. $\mathbf{w}=\left[w_{1}, \ldots, w_{m}\right]^{\top}$ is a vector of coefficients. $b$ is the bias which is defined as the mean of $\sum_{j=1}^{n} \sum_{i=1}^{m} \kappa\left(\mathbf{x}_{i}, \mathbf{x}_{j}\right) w_{i}$ [8], [16]. The kernel function $\kappa$ is defined as

$$
\kappa\left(\boldsymbol{x}_{i}, \boldsymbol{x}_{j}\right)=\exp \left(-\left(d\left(\boldsymbol{x}_{i}, \boldsymbol{x}_{j}\right)\right)^{2} / 2 \sigma^{2}\right)
$$

where $\sigma$ is used to scale the exponential function. It derives a nonlinear model by mapping the data points to an infinite dimensional Hilbert space, which is more appropriate for the nonlinear dataset [8]. For convenience, when we have $r$ hash bits for each sample, the hash function can be written as

$$
\mathbf{y}_{i}=\operatorname{sgn}\left(\mathbf{z}_{i}^{\top} \mathbf{W}\right)
$$

where $\mathbf{y}_{i}=\left[y_{1}, \ldots, y_{r}\right]$ is the hash code of sample $\mathbf{x}_{i}$, and $\mathbf{z}_{i}$ is calculated as

$$
\mathbf{z}_{i}=\left[\kappa\left(\mathbf{x}_{1}, \mathbf{x}_{i}\right)-\mu_{1}, \kappa\left(\mathbf{x}_{2}, \mathbf{x}_{i}\right)-\mu_{2}, \ldots, \kappa\left(\mathbf{x}_{m}, \mathbf{x}_{i}\right)-\mu_{m}\right]^{\top}
$$

where

$$
\mu_{i}=\sum_{j=1}^{n} \kappa\left(x_{j}, x_{i}\right) / n
$$

and

$$
\mathbf{W}=\left[\mathbf{w}_{1}, \ldots, \mathbf{w}_{r}\right]
$$

In the prediction function, $\mathbf{W}$ defines the hash functions so that we need to learn $\mathbf{W}$ with supervised hierarchical information.

\section{B. Semantic Level Learning}

In many supervised hashing research [26], [23], pairwise labels are given as supervised information for training. When a pair of samples are similar, their pairwise label is 1 , otherwise it is -1 . The similarity between pairs may be acquired from their semantic relationship. However, most semantic information correspond to flat relationship. When structural semantic relationship is available on the dataset such as tree structure of the ImageNet, merely using -1 and 1 to reveal the pairwise 
relationship seems to be insufficient. Here we define a label matrix $\mathbf{M}_{i j}$ to reflect the relationship of all samples. The distance between two samples can be defined as

$$
\operatorname{dis}\left(\mathbf{x}_{i}, \mathbf{x}_{j}\right)=h\left(\mathbf{x}_{i}, \mathbf{x}_{j}\right) / h^{*}
$$

where $h\left(\mathbf{x}_{i}, \mathbf{x}_{j}\right)$ is the hierarchical distance, defined as the number of levels to the lowest common ancestor of $\mathbf{x}_{i}$ and $\mathbf{x}_{j}$, e.g. dis $(E-$ guitar, $C-$ guitar $)$ is 1 in Figure I, and $h^{*}$ is the height of the tree. We not only compute the height of the lowest common ancestor of two samples but also take the height of the tree they belong to into consideration. This is the normalized height that can better reflect the sample relationship. When the pairwise distances between all samples are generated, we can calculate an appropriate value to reveal the pairwise relations according to the distances. Since many works have used 1 and -1 to define the similarity, a value between -1 and 1 is preferred and can be defined as

$$
g\left(\operatorname{dis}\left(\mathbf{x}_{i}, \mathbf{x}_{j}\right)\right)=1-\gamma \operatorname{dis}\left(\mathbf{x}_{i}, \mathbf{x}_{j}\right)
$$

where $\gamma$ is a parameter to control the contribution of the pairwise distance. Then the label matrix $\mathbf{M}$ can be calculated as

$$
\mathbf{M}_{i j}=\left\{\begin{array}{lc}
g\left(\operatorname{dis}\left(\mathbf{x}_{i}, \mathbf{x}_{j}\right)\right), & \text { if }\left(\mathbf{x}_{i}, \mathbf{x}_{j}\right) \in T \\
-1, & \text { otherwisie }
\end{array}\right.
$$

where $\left(\mathbf{x}_{i}, \mathbf{x}_{j}\right) \in T$ means $\mathbf{x}_{i}$ and $\mathbf{x}_{j}$ belong to the same tree. 1 . When $\mathbf{x}_{i}$ and $\mathbf{x}_{j}$ have no common parent in the hierarchical dataset, $\mathbf{M}_{i j}$ is -1 which suggests they are not related. When they have a common parent, $\mathbf{M}_{i j}$ is based on the distance between them in the hierarchical structure.

Since a node in the tree structure only has binary codes, the hash functions are only learned for images. We can learn hash functions by leveraging hierarchical supervised information, so that the generated hash codes of each sample reflect the hierarchical structure of the dataset as shown in Figure I. On one hand, the codes of nodes in different trees have larger Hamming distances in the binary space. On the other hand, in the same tree, the codes of nodes have longer common subsequence but are still different from each other. Inspired by the work of Liu et al. [26] that minimizes the code inner products and the Hamming distances, we define the objective function by incorporating the label matrix $\mathbf{M}$ as follows:

$$
\mathbf{E}_{\mathbf{W}}=\left\|\frac{1}{r} \mathbf{Y} \mathbf{Y}^{\top}-\mathbf{M}\right\|_{F}^{2}
$$

$\mathbf{M}$ is the label matrix and $\mathbf{Y}$ is defined as follows

$$
\mathbf{Y}=\left[\begin{array}{ccc}
\mathbf{y}_{1}\left(\mathbf{x}_{1}\right) & \ldots & \mathbf{y}_{r}\left(\mathbf{x}_{1}\right) \\
\mathbf{y}_{1}\left(\mathbf{x}_{2}\right) & \ldots & \mathbf{y}_{r}\left(\mathbf{x}_{2}\right) \\
\ldots \ldots & & \\
\mathbf{y}_{1}\left(\mathbf{x}_{n}\right) & \ldots & \mathbf{y}_{r}\left(\mathbf{x}_{n}\right)
\end{array}\right]=\operatorname{sgn}\left(\mathbf{Z}^{\top} \mathbf{W}\right)
$$

where $\mathbf{Z}=\left[\mathbf{z}_{1}, \ldots, \mathbf{z}_{n}\right]$ and $\mathbf{W}=\left[\mathbf{w}_{1}, \ldots, \mathbf{w}_{r}\right]$. Eq. (10) guarantees that the hash codes of each sample match its semantic relationship in the data hierarchy because both semantic and feature level relationships have been taken into consideration. In the next section, we analyze the feature level relationship.

\footnotetext{
${ }^{1}$ ImageNet is decomposed to some subtrees, and the reason is given in Section IV
}

\section{Feature Level Learning}

Hierarchical image retrieval requires binary codes for each node in the tree structure. Although hash codes for image samples can be calculated using hash functions, it is not straightforward to generate the hash codes for the nodes because they have category labels rather than vectorized data. Inspired by the hash clustering method proposed by Gong et al. [37], we propose to calculate each hash codes of node by binary clustering the hash codes of training samples belonging to this node. Assuming we have $r$ bit hash codes for data and nodes, the objective function is

$$
\begin{aligned}
& \min _{\mathbf{c}} \sum_{i=1}^{p}\left\|\mathbf{y}_{i}^{\top}-\mathbf{c}\right\|_{2}^{2} \\
& \text { s.t. } \quad \mathbf{c} \in\{-1,+1\}^{1 \times r} .
\end{aligned}
$$

where $p$ is the number of training samples in the cluster, $\mathbf{y}_{i}$ is the binary hash codes of sample $x_{i}$, and $\mathbf{c} \subset \mathbb{R}^{r}$ is the center of the cluster. Then $\mathbf{c}$ is treated as the hash codes of the node. Note that $p$ varies with the structure of the dataset. When the codes of leaf nodes are obtained, we can calculate the codes of their father node. Step by step, we can obtain the codes of all nodes. Let $\mathbf{C}=\left[\mathbf{c}_{1}, \ldots, \mathbf{c}_{q}\right] \subset \mathbb{R}^{r \times q}$ be the binary codes of nodes to be learned, where the number of nodes in the tree structure is $q$, all $\mathbf{c}$ must be restrained by

$$
\mathbf{E}_{\mathbf{C}}=\sum_{l=1}^{q} \sum_{i=1}^{p}\left\|\mathbf{y}_{i}^{\top}-\mathbf{c}_{l}\right\|_{2}^{2}
$$

Minimizing Eq. (13) guarantees that each cluster center or node be close to its children at the feature level. Therefore, the hash codes of the father node and the hash codes of its children nodes have short Hamming distance (see the example of hash codes in Figure (I)). In this way, when we have the hash codes of training samples, we can estimate the hash codes of all nodes throughout the whole hierarchical structure by Eq. (13). Our optimization method guarantees the feature level relationship of these nodes.

\section{Final Objective Function}

Combining semantic level and feature level objective functions, the final objective function to be minimized is

$$
\begin{aligned}
\mathbf{E}= & \mathbf{E}_{\mathbf{W}}+\lambda \mathbf{E}_{\mathbf{C}} \\
= & \left\|\frac{1}{r} \operatorname{sgn}\left(\mathbf{Z}^{\top} \mathbf{W}\right) \operatorname{sgn}\left(\mathbf{Z}^{\top} \mathbf{W}\right)^{\top}-\mathbf{M}\right\|_{F}^{2} \\
& +\lambda \sum_{l=1}^{q} \sum_{i=1}^{p_{c}}\left\|\operatorname{sgn}\left(\mathbf{z}_{i}^{\top} \mathbf{W}\right)-\mathbf{c}_{l}\right\|_{2}^{2}
\end{aligned}
$$

where the first term is from Eq. (10) and the second term is from Eq. (13). In the second term, we minimize the distance between every node and their children nodes in the hierarchical structure $^{2} . \lambda$ is a parameter to balance the contribution of two terms. When $\lambda$ is small, the contribution of first term increases so that the whole function is more likely to follow the semantic relationship of the hierarchical structure since $\mathbf{M}$ models the

\footnotetext{
${ }^{2}$ The children of the leaf nodes are the training image samples.
} 
semantic relationship. When $\lambda$ is large, it is more likely to guarantee the feature level relationship since more weights are assigned to minimize the distances between samples and their cluster center. The model reduces to traditional flat hashing when $\lambda$ is set to 0 . The goal here is to estimate $\mathbf{W}$ by minimizing Eq. (14). In the next subsection, the optimization method is discussed.

\section{E. Optimization}

After some mathematical deductions, optimization of Eq. (14) can be re-written as

$$
\begin{gathered}
\min _{\mathbf{w}_{k}, c_{l_{k}}}\left\|\sum_{k=1}^{r} \operatorname{sgn}\left(\mathbf{Z}^{\top} \mathbf{w}_{k}\right) \operatorname{sgn}\left(\mathbf{Z}^{\top} \mathbf{w}_{k}\right)^{\top}-r \mathbf{M}\right\|_{F}^{2} \\
+\lambda r^{2} \sum_{l=1}^{q} \sum_{i=1}^{p_{c}} \sum_{k=1}^{r}\left\|\operatorname{sgn}\left(\mathbf{z}_{i}^{\top} \mathbf{w}_{k}\right)-c_{l_{k}}\right\|_{2}^{2}
\end{gathered}
$$

where $\mathbf{w}_{k}(k=1, \ldots, r)$ are separated from $\mathbf{W}$ in Eq. 14 , and $c_{l_{k}}$ is the is the $k$-th entry of $\mathbf{c}_{l}$. There are two terms to be optimized, $\mathbf{W}$ and $\mathbf{c}$. We alternatively optimize the objective function in $r$ iterations. In each iteration, we only solve $\mathbf{w}_{k}$ and $c_{l_{k}}$ which generates the $k$-th bit code of all the cluster centers given the previously solved vectors $\mathbf{w}_{1}^{*}, \ldots, \mathbf{w}_{k-1}^{*}$ and $\mathbf{c}_{l_{1}}^{*}, \ldots, \mathbf{c}_{l_{k-1}}^{*}(l=1, \ldots, q)$.

1) Optimize $\mathbf{W}$ : In each iteration of optimization, we first optimize $\mathbf{w}_{k}$ with fixed $c_{l_{k}}(l=1, \ldots, q)$, which is the $k$-th dimension of the codes of all the cluster centers. Let

$$
T_{k-1}=r M-\sum_{t=1}^{k-1} \operatorname{sgn}\left(\mathbf{Z}^{\top} \mathbf{w}_{t}^{*}\right) \operatorname{sgn}\left(\mathbf{Z}^{\top} \mathbf{w}_{t}^{*}\right)^{\top}
$$

where $T_{0}=r M$, then we can minimize the following cost function

$$
\begin{aligned}
& \left\|\operatorname{sgn}\left(\mathbf{Z}^{\top} \mathbf{w}_{k}\right) \operatorname{sgn}\left(\mathbf{Z}^{\top} \mathbf{w}_{k}\right)^{\top}-T_{k-1}\right\|_{F}^{2} \\
& +\lambda \sum_{l=1}^{q} \sum_{i=1}^{p_{c}} \sum_{k=1}^{r}\left\|\operatorname{sgn}\left(\mathbf{z}_{i}^{\top} \mathbf{w}_{k}\right)-c_{l_{k}}\right\|_{2}^{2} \\
= & \left(\operatorname{sgn}\left(\mathbf{Z}^{\top} \mathbf{w}_{k}\right)\left(\operatorname{sgn}\left(\mathbf{Z}^{\top} \mathbf{w}_{k}\right)\right)^{\top}\right)^{2} \\
& -2\left(\operatorname{sgn}\left(\mathbf{Z}^{\top} \mathbf{w}_{k}\right)\right)^{\top} T_{k-1} \operatorname{sgn}\left(\mathbf{Z}^{\top} \mathbf{w}_{k}\right)+\operatorname{tr}\left(T_{k-1}^{2}\right) \\
& +\lambda r^{2} \sum_{l=1}^{q} \sum_{i=1}^{p_{c}} \sum_{k=1}^{r}\left(\operatorname{sgn}\left(\mathbf{z}_{i}^{\top} \mathbf{w}_{k}\right)\right)^{2}+\lambda r^{2} \sum_{l=1}^{q} \sum_{i=1}^{p_{c}} \sum_{k=1}^{r} c_{l_{k}}^{2} \\
& -2 \lambda r^{2} \sum_{l=1}^{q} \sum_{i=1}^{p_{c}} \sum_{k=1}^{r} \operatorname{sgn}\left(\mathbf{z}_{i}^{\top} \mathbf{w}_{k}\right) c_{l_{k}} \\
= & \operatorname{const}-2 \lambda r^{2} \sum_{l=1}^{q} \sum_{i=1}^{p_{c}} \sum_{k=1}^{r} \operatorname{sgn}\left(\mathbf{z}_{i}^{\top} \mathbf{w}_{k}\right) c_{l_{k}} \\
& -2 \operatorname{sgn}\left(\mathbf{Z}^{\top} \mathbf{w}_{k}\right)^{\top} T_{k-1} \operatorname{sgn}\left(\mathbf{Z}^{\top} \mathbf{w}_{k}\right)
\end{aligned}
$$

Discarding the constant term, we get

$$
\begin{gathered}
\phi\left(\mathbf{w}_{k}\right)=-\operatorname{sgn}\left(\mathbf{Z}^{\top} \mathbf{w}_{k}\right)^{\top} T_{k-1} \operatorname{sgn}\left(\mathbf{Z}^{\top} \mathbf{w}_{k}\right) \\
-\lambda r^{2} \sum_{l=1}^{q} \sum_{i=1}^{p_{c}} \sum_{k=1}^{r} \operatorname{sgn}\left(\mathbf{z}_{i}^{\top} \mathbf{w}_{k}\right) c_{l_{k}}
\end{gathered}
$$

Since $\operatorname{sgn}($.$) function is difficult to process, we replace it$ with a sigmoid-shaped function

$$
\varphi(a)=\frac{2}{1+e^{-a}}-1
$$

which is sufficiently smooth and is an approximation of $\operatorname{sgn}($.$) . Then Eq. (18) can be written as$

$$
\begin{gathered}
\widetilde{\phi}\left(\mathbf{w}_{k}\right)=-\varphi\left(\mathbf{Z}^{\top} \mathbf{w}_{k}\right)^{\top} T_{k-1} \varphi\left(\mathbf{Z}^{\top} \mathbf{w}_{k}\right) \\
-\lambda r^{2} \sum_{l=1}^{q} \sum_{i=1}^{p_{c}} \sum_{k=1}^{r} \varphi\left(\mathbf{z}_{i}^{\top} \mathbf{w}_{k}\right) c_{l_{k}}
\end{gathered}
$$

The gradient of $\widetilde{\phi}$ with respect to $w_{k}$ is

$$
\begin{aligned}
\nabla \widetilde{\phi}= & -\mathbf{Z}\left(\left(T_{k-1} \varphi\left(\mathbf{Z}^{\top} \mathbf{w}_{k}\right)\right) *\left(\mathbf{1}-\varphi\left(\mathbf{Z}^{\top} \mathbf{w}_{k}\right) * \varphi\left(\mathbf{Z}^{\top} \mathbf{w}_{k}\right)\right)\right) \\
& -\lambda r^{2} \sum_{l=1}^{q} \sum_{i=1}^{p_{c}} \sum_{k=1}^{r} c_{l_{k}} \mathbf{z}_{i} *\left(\mathbf{1}-\varphi\left(\mathbf{z}_{i}\right) * \varphi\left(\mathbf{z}_{i}\right)\right)
\end{aligned}
$$

where the symbol $*$ represents the Hadamard product and $\mathbf{1}$ is all one vector. Then $\mathbf{w}$ can be optimized by the gradient descent method [38].

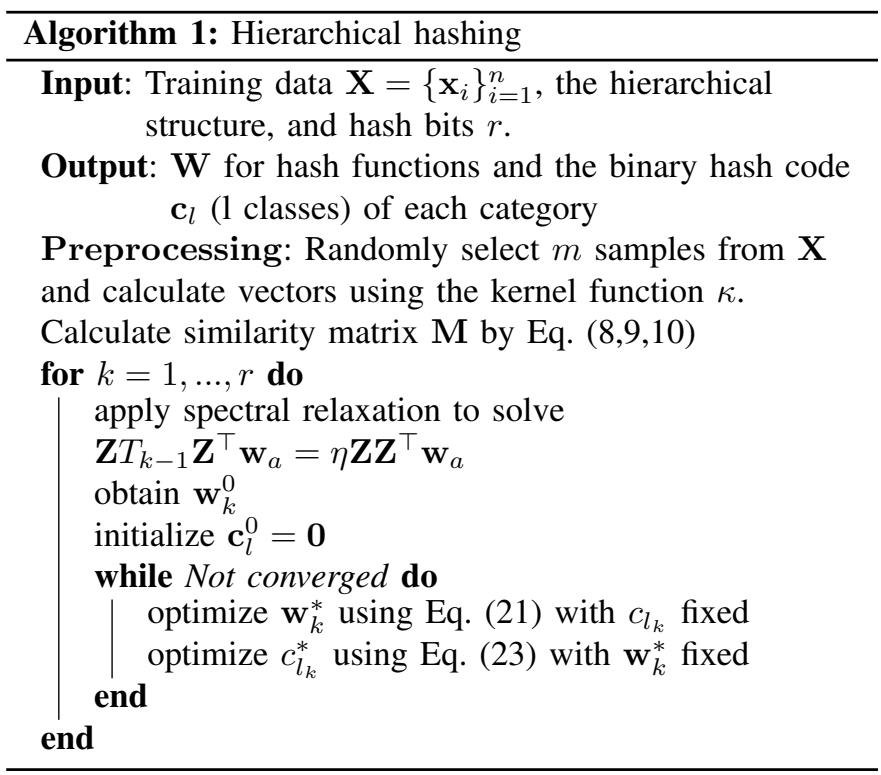

2) Optimize c: We fix $\mathbf{w}_{k}$ to optimize $c_{l_{k}}$. Discarding the constant term and the terms that do not contain c, Eq. (17) can be written as

$$
\min _{c_{l_{k}}}-2 \lambda r^{2} \sum_{l=1}^{q} \sum_{i=1}^{p_{c}} \sum_{k=1}^{r} \operatorname{sgn}\left(\mathbf{z}_{i}^{\top} \mathbf{w}_{k}\right) c_{l_{k}}
$$

Since $c_{l_{k}} \in\{-1,1\}$, minimizing Eq. (22) leads to

$$
\psi=\operatorname{sgn}\left(\lambda \sum_{l=1}^{q} \sum_{i=1}^{p_{c}} \operatorname{sgn}\left(\mathbf{z}_{i}^{\top} \mathbf{W}\right)^{\top}\right)
$$

When $\mathbf{w}_{k}$ has been generated from Section III-E1, we can directly calculate the $k$-th code $c_{l_{k}}$ for the center $\mathbf{c}_{\mathbf{l}}$. With the new $c_{l_{k}}$, we can continue to optimize $\mathbf{w}_{k}$. This updates 


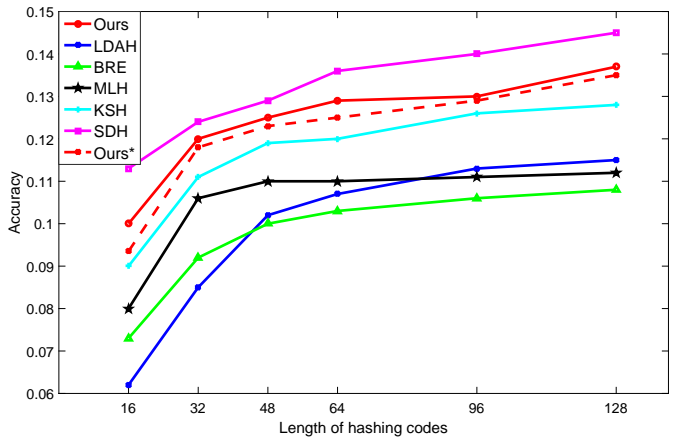

Result on ImageNet

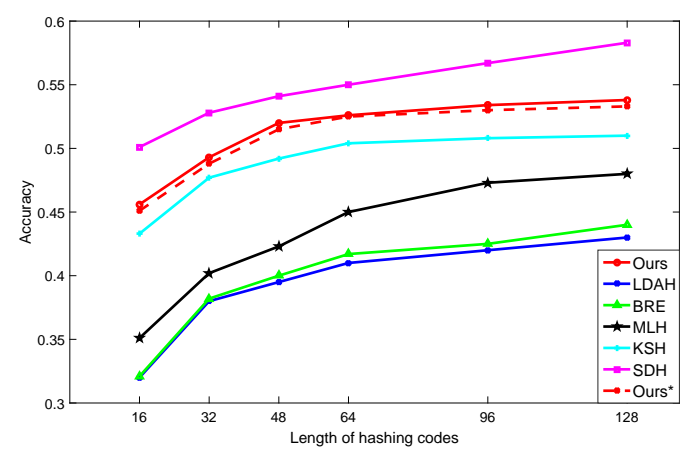

Result on AWA

Fig. 2. The result of top-20 retrieved images using different number of bit codes with the state-of-art methods on Imagenet and AWA datasets.

W so that $c_{l_{k}}$ can be optimized again. $\mathbf{w}_{k}$ and $c_{l_{k}}$ are optimized iteratively until the change of Eq. (15) is lower than a threshold. This completes the optimization for $\mathbf{w}_{k}$ and $c_{l_{k}}$ $(k=1, \ldots, r)$. For $r$ bits, optimal $\mathbf{W}$ and $\mathbf{c}_{l}(l=1, \ldots, q)$ can be generated after $r$ iterations. In the first iteration to estimate $\mathbf{w}_{k}$, we keep the first term of Eq. (15) unchanged and apply spectral relaxation by dropping the $\operatorname{sgn}($.$) term to compute$ $\mathbf{w}_{k}^{0}$ for initialization of $\mathbf{w}_{k}$

$$
\min _{\mathbf{w}_{k}}-\left(\mathbf{Z}^{\top} \mathbf{w}_{k}\right)^{\top} T_{k-1}\left(\mathbf{Z}^{\top} \mathbf{w}_{k}\right)
$$

which is similar to the method by Liu et al. [26]. This is a standard eigenvalue problem, i.e., $\mathbf{Z} T_{k-1} \mathbf{Z}^{\top} \mathbf{w}_{k}=\eta \mathbf{Z} \mathbf{Z}^{\top} \mathbf{w}_{k}$, where $\mathbf{w}_{k}$ is sought as the eigenvector associated with the largest eigenvalue. With $\mathbf{w}_{k}$ estimated, we can calculate $c_{l_{k}}$ corresponding to $\mathbf{w}_{k}$, which is brought into the optimization function as the input to the initial round of calculation. A summary of our method is shown in Algorithm 1.

The time complexity for training stage is $O\left(\left(n m t_{w}+\right.\right.$ $\left.p q) t_{r} r\right)$ where $n \gg p, n \gg q, n \gg m, t_{w}$ is the number of iterations for $\mathbf{w}_{k}$, and $t_{r}$ is the number of iterations for each $r$ bits. The time complexity for testing stage is constant $O(m r d)$.

\section{EXPERIMENTS}

We evaluated the effectiveness of the hierarchical hashing method on two hierarchical image datasets. Three experiments were performed on two vision tasks: image retrieval and classification.

\section{A. Datasets}

The first dataset is ImageNet ILSVRC2010 [29]. It is a subset of ImageNet which is organized according to the WordNet hierarchy. It contains about 1.2 million images belonging to 1,000 classes. A tree structure can be generated from the dataset, in which all 1,000 classes are considered as the leaf nodes whose children are images in the classes and the higher level nodes contain multiple categories in their corresponding children nodes. SIFT-based bag-of-words features were extracted to represent each image [39], so that each image was represented as a vector of 1,000 dimensions.

We did not used the whole ImageNet dataset in the experiments because many image classes do not have multichildren hierarchical structures. They have only one node on each layer and only the leaf node contains images as displayed in part $\mathrm{A}$ of Figure 3. This makes the subtree equivalent to flat structure. Unlike part A, part B has a multi-children hierarchical structure. Each node of this part has some children and these relationship information is significantly important since the images belong to the same category look like each other so their features are similar. In the experiments, we only used classes with more complex hierarchical structure.

The second dataset is Animals with Attributes (AWA) dataset [40] which contains 30475 images in 50 animals classes. The features adopted in our experiments were pre-trained using a seven layer CaffeNet [41]. Each image was represented by a vector of 4,096 dimensions. In the experiment, we used five subtrees of ImageNet and built a tree structure for the AWA dataset with the hierarchical relationship according to the WordNet hierarchy. The details are described in the corresponding experiments.

\section{B. Flat Hash Retrieval}

In the first experiment, we compared our method with several state-of-art supervised hashing methods on traditional flat hash retrieval tasks. Image data were retrieved directly at the bottom level without going through the hierarchy, following how most existing hashing methods work. Note that the training of our method still requires hierarchical information, but our method is capable of performing traditional retrieval. The methods included in the comparison were supervised discrete hashing (SDH) [28], LDAhash (LDAH) [21], learning to hash with binary reconstructive embeddings (BRE) [32], minimal loss hashing for compact binary codes (MLH) [23], and supervised hashing with kernels (KSH) [26]. SDH is a hashing framework where the objective is to learning hash codes for classification. LDAH is a supervised approach that integrates an easy optimization process. BRE and MLH require complex optimization but have higher search accuracy. KSH has relatively simple optimization with good performance.

In the experiments, the same kernel $\kappa\left(x_{i}, x_{j}\right)=$ $\exp \left(-\left\|x_{i}-x_{j}\right\|^{2} / \sigma^{2}\right)$ was used for each kernel method where parameter $\sigma$ was tuned to an appropriate value on each dataset. 

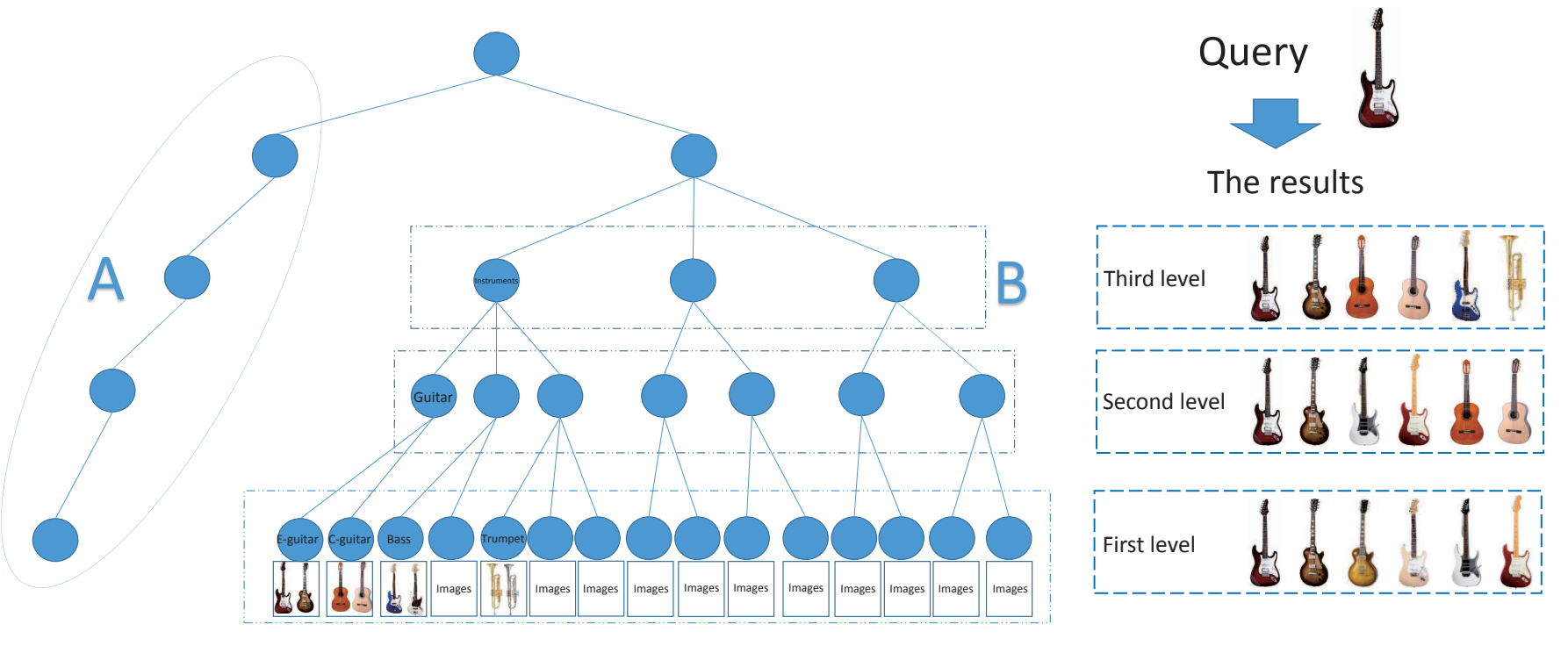

Fig. 3. Subtree B on the right has complex hierarchical structure, but subtree A does not. With respect to hierarchical retrieval we build multiple hash tables for different correlation levels of the retrieval results. The right part is a sketch map to show the results in different levels for hierarchical retrieval.
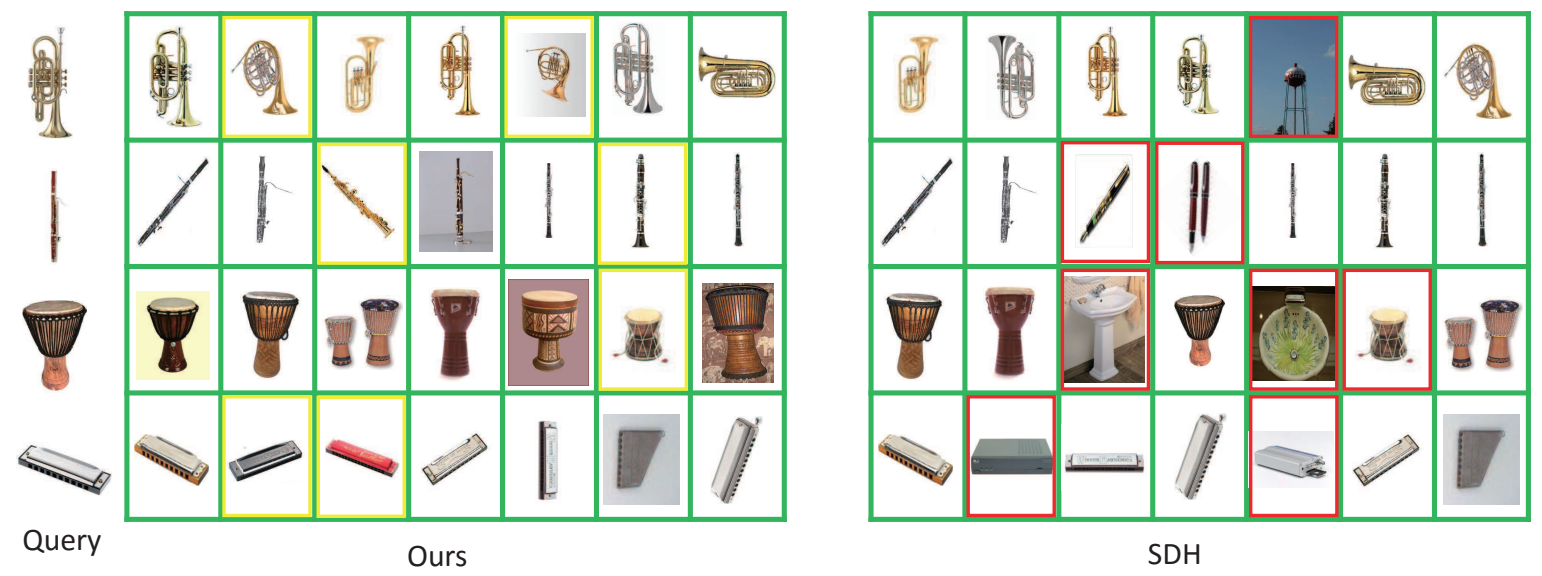

Fig. 4. A example of the retrieval results with respect to different methods with 128 bits. The left is the results of the proposed method and the right is results of SDH. As is shown on the left, the retrieval results in yellow boxes have common father with the others in green boxes. On the contrary, with respect to the right part, the wrong results in red boxes have no common ancestor with the right ones, let alone same father.

We used the publicly available codes of these methods and the parameters were set to the suggested values (if given). We selected 502 classes in ImageNet ILSVRC2010 for this experiment. For the AWA, the whole dataset was used. We randomly sampled 70 percent images to form the training set and took the rest images for testing. Furthermore, to show the performance of our method with a small training set, we also used 100 training images for our method.

The retrieved samples were ranked based on their Hamming distances to the query. In the experiments, we calculated the mean average precision of retrieval based on the top 20 retrieved images. The changes on accuracy with respect to different length of hash codes were adopted to evaluate the performance of different methods. The accuracy are shown in Figure 2. Red line named Ours* is the result of our method by using 100 training images for each class, while all other methods were trained with all training images for each class. It can be seen from the figure that our method is better than the other methods except SDH in terms of accuracy. SDH has the best performance on flat retrieval, however, for the hierarchical retrieval our method is significantly better than SDH (see Section IV-C). The experiment is to prove that our method can handle and achieve good performance on the flat retrieval problem. We can also see that, the proposed method 


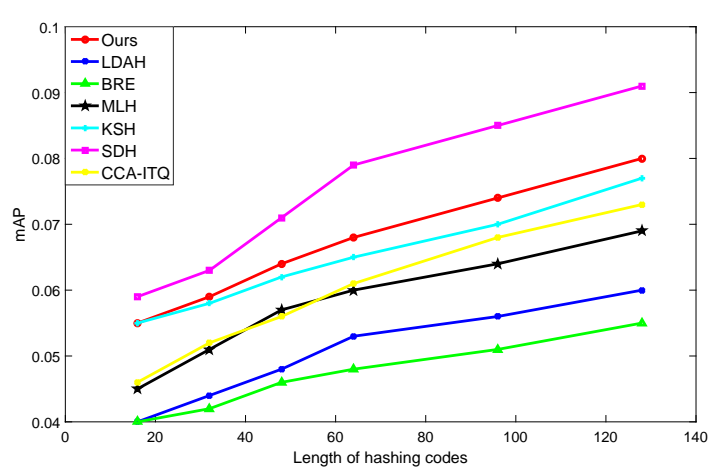

(A) The first level

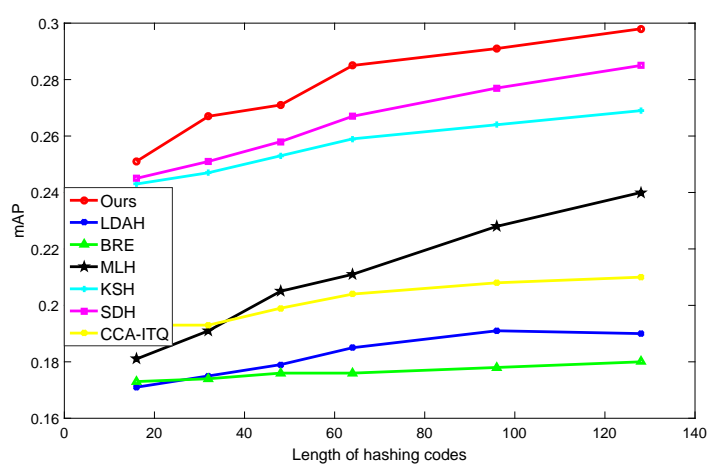

(C) The third level

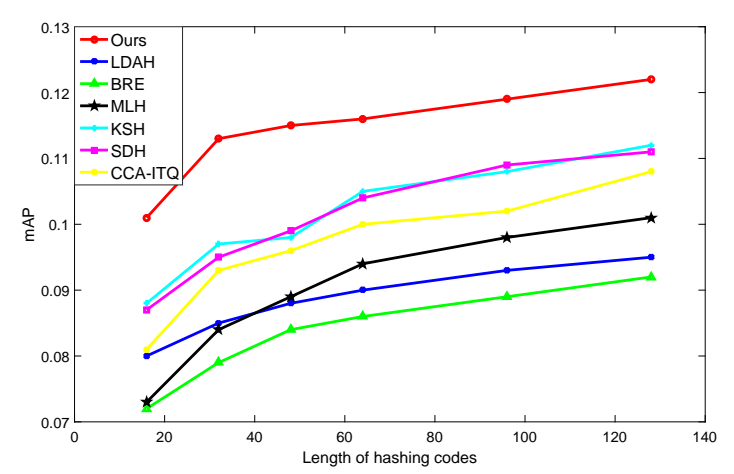

(B) The second level

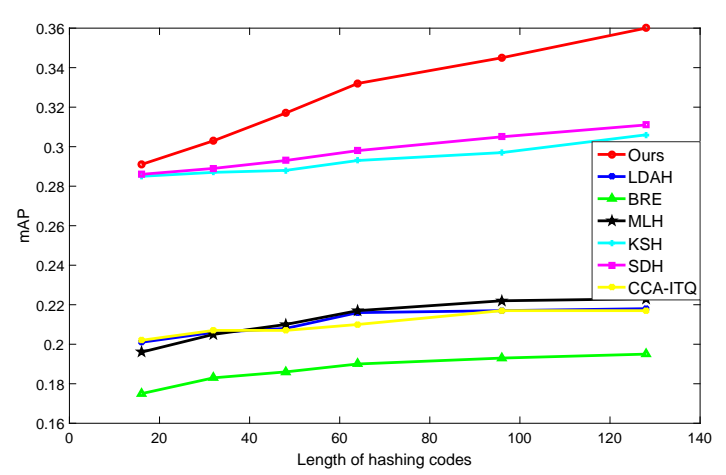

(D) The fourth level

Fig. 5. mAPs of different methods with varying code lengths for different levels on ImageNet dataset.

also has good performance with a small training set so that the training time can be significantly reduced. Among the alternative methods, SDH is the best in terms of accuracy, outperforming LDAH, BRE, MLH, and KSH with a large margin. Furthermore, we show a example of retrieval results in Figure 4. The proposed method already achieves good performance, besides, the retrieval results is more a reflection of the hierarchical information. As shown on the left part, for each line, the retrieval results in yellow boxes have common father with the others in green boxes, which means they belong to the same class on the high hierarchical level. On the contrary, as shown in the right, the wrong results in red boxes have no common ancestor with the right ones, let alone same father. Since we leverage hierarchical information, we can guarantee the results that are close to the query as much as possible. The time for training and testing are shown in Table I.

\section{Hierarchical Hash Retrieval}

In our method, each category in the taxonomy structure of the dataset is a node and has a hash code. To provide evaluation of different methods on the hierarchical retrieval task, we present a novel hierarchical search strategy here. In this strategy, we have multiple hash tables, the number of which equals the maximum depth of the trees. We can select

\begin{tabular}{|c|c|c|c|c|c|}
\hline Method & Ours & SDH & BRE & MLH & KSH \\
\hline \hline Training Time(s) & 3101 & 11 & 10865 & 2035 & 2561 \\
\hline Testing Time(s) & $2 \mathrm{e}-6$ & $2 \mathrm{e}-6$ & $5 \mathrm{e}-5$ & $2 \mathrm{e}-5$ & $2 \mathrm{e}-6$ \\
\hline
\end{tabular}

TABLE I

TRAINING AND TESTING TIME OF VARIOUS METHODS WITH 48 BITS ON AWA DATASET. WE USE A DESKTOP PC WITH AN INTEL QUAD-CORE 4.0GHZ CPU AND 128G RAM

different levels of retrieval results. For instance, as is shown in Figure 3, given an E-guitar as a query, when we select the first level, i.e., the leaf node level which is the most accurate, the results are supposed to be formed by all E-guitar images. When we use the second level, the results may be formed by E-guitar and C-guitar. When we select the third level, the results may contain E-guitar, C-guitar and some other musical instruments such as Bass, and therefore, are less accurate. For each level, we build a hash table, in which we can replace the code of each image with the code of the node it belongs to in the binary space by calculating their hamming distances. Our model can provide an optional search strategy for different targets and most important of all, it requires only one time of training.

We also used 502 classes with hierarchical structure for 


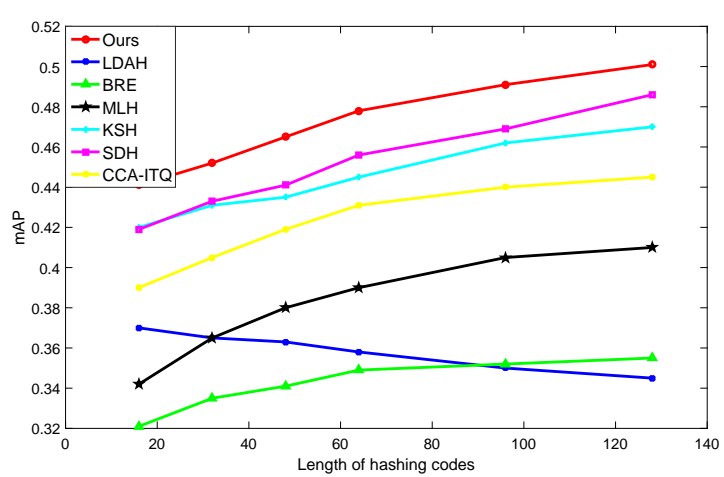

Second level

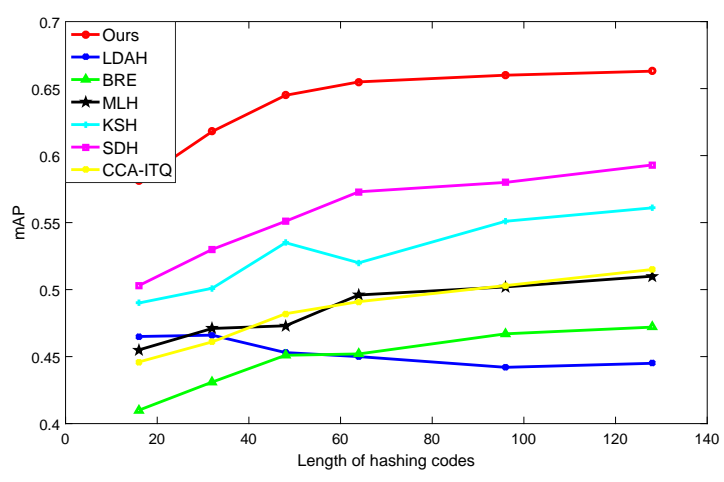

Third level

Fig. 6. The mAPs of different methods with varying code lengths for the second and third levels on the AWA dataset.

this experiment. We randomly selected 1000 samples in each class for training and the rest samples for testing. The max depth of these subtrees of ImageNet was 4 , so we built 4 hash tables for retrieval. All the compared methods, including LDAhash (LDAH) [21], learning to hash with binary reconstructive embeddings (BRE) [32], minimal loss hashing for compact binary codes (MLH) [23], iterative quantization (CCA-ITQ) [20], supervised hashing with kernels (KSH) [26], and supervised discrete hashing (SDH) [28], were trained with the labels in the first level (leaf node level). With respect to retrieval, for different levels, the labels of both training and testing images in the compared method were replaced by the labels of nodes in the corresponding levels. The results are displayed in Figure 5.

With respect to the AWA dataset, we built a tree structure according to the WordNet hierarchy. The structure included 3 levels with 16 classes in the leaf nodes. The labels of all images were also replaced by the labels of cluster centers (nodes) in the corresponding level. The number of training samples in each class was 70 percent and the rest were used for testing. The results are shown in Figure 6.

It is clear that our method has significantly outperformed all other approaches in high levels. Since the retrieval on the first level is equivalent to flat retrieval, the mAP of SDH is higher than ours. However, our method can better deal

\begin{tabular}{|c|c|c|c|c|}
\hline Subtree & Train & Test & Class & Depth \\
\hline Music & 2500 & 1250 & 25 & 6 \\
\hline Furniture & 2300 & 1150 & 23 & 4 \\
\hline Tool & 2600 & 1300 & 26 & 6 \\
\hline Amphibian & 800 & 400 & 8 & 4 \\
\hline Geo & 1200 & 600 & 12 & 5 \\
\hline AWA & 1600 & 800 & 16 & 3 \\
\hline
\end{tabular}

TABLE II

SUBTREES EXTRACTED FROM THE IMAGENET DATASET FOR CLASSIFICATION PERFORMANCE EVALUATION.

with hierarchical retrieval especially on high levels with one time of training. We use the structure relationship of the classes in the learning process, which guarantees that the classes belonging to the same father node have short Hamming distances than the classes that have no common father node. These results indicate that our method ensures the retrieval results by including images not only with similar features as the query but also close to the query in the dataset hierarchy.

\section{Results on Classification}

The purpose of this experiment was to evaluate the effectiveness of the intermediate nodes in the classification task. The experiments were performed on a subset of ImageNet containing 6 subtrees whose depth and the number of classes was shown in Table II. The length of hash code was set to 256 . The evaluation criterion for classification shall be able to reflect the effectiveness of hierarchical hashing.

To this end, we use both flat error and hierarchical error to compute the error rates. Given an image, if the predicted class is its true class, the flat error is 0 , otherwise, the flat error is 1. The hierarchical error reports the distance from the lowest common ancestor to the level of predicted class and its true class. For example, in Figure I, if the query is Trombone and the predicted class is E-Guitar, the hierarchical error is 2 since their lowest common ancestor is Instrument. This criterion has been used by Zhao et al. [31] to evaluate hierarchical feature hashing method.

The node classification process was as follows. We first compared the hash codes of a query with the root node of each tree, then compared it to the children of the root node, and moved to the child that had the shortest Hamming distance with the query. We repeated this process until the query image was classified into a leaf node. We compared our method with several classification methods which used image feature to train classifiers. These methods included multi-class SVM (MSVM) [42], hierarchical SVM (HieSVM) [43], tree classifier (TreeSVM) [44] which trained a multi-class SVM at each node, and HESHING [31] which used hash function for each class.

Table III shows the experimental results. The results show that our hierarchical hashing method has comparable classification performance as the alternative approaches. With 


\begin{tabular}{|c|c|c|c|c|c|c|c|}
\hline \multicolumn{2}{|c|}{ Subtree } & Music & Fur & Tool & Amp & Geo & AWA \\
\hline \multicolumn{2}{|c|}{ Depth } & 6 & 4 & 6 & 4 & 5 & 3 \\
\hline \multirow{2}{*}{ HieSVM } & F Error & 0.80 & 0.58 & $\mathbf{0 . 7 2}$ & 0.64 & $\mathbf{0 . 5 0}$ & 0.45 \\
& H Error & 4.42 & 2.16 & $\mathbf{3 . 7 6}$ & 1.86 & 2.14 & 1.62 \\
\hline \multirow{2}{*}{ TreeSVM } & F Error & 0.82 & 0.64 & 0.86 & 0.70 & 0.52 & 0.43 \\
& H Error & 4.82 & 3.16 & 5.54 & 2.42 & 2.36 & 1.78 \\
\hline \multirow{2}{*}{ HESHING } & F Error & $\mathbf{0 . 7 6}$ & $\mathbf{0 . 5 4}$ & 0.74 & 0.60 & 0.50 & 0.41 \\
& H Error & 4.12 & 2.34 & 3.96 & 1.82 & 2.18 & $\mathbf{1 . 4 1}$ \\
\hline \multirow{2}{*}{ MSVM } & F Error & 0.80 & 0.61 & 0.79 & 0.62 & 0.50 & 0.44 \\
& H Error & 4.60 & 2.75 & 4.89 & 1.94 & 2.24 & 1.75 \\
\hline \multirow{2}{*}{ Ours } & F Error & 0.81 & 0.61 & 0.73 & $\mathbf{0 . 5 9}$ & 0.51 & $\mathbf{0 . 4 0}$ \\
& H Error & $\mathbf{3 . 8 0}$ & $\mathbf{1 . 9 8}$ & 3.80 & $\mathbf{1 . 8 1}$ & $\mathbf{2 . 0 5}$ & 1.43 \\
\hline
\end{tabular}

TABLE III

CLASSIFICATION RESULTS FROM VARIOUS METHODS ON IMAGENET.

the information of class taxonomy, our method has achieved better results on some subtrees of the dataset, especially for the hierarchy error. By keeping the critical information and the relationship of the hierarchical structure of dataset, our method gets benefit in achieving lower errors. In particular, the effectiveness of the node codes has been well-proven in this experiment.

\section{CONCLUSIONS}

In this paper, a hierarchical hashing method for image retrieval has been introduced. This method takes both hierarchical semantic level relationship and feature level relationship of images into consideration and fuses them to a learning objective. The proposed method has been evaluated using both general evaluation criteria and specific hierarchical criteria on the hierarchical databases. The experimental results on two hierarchical datasets show that our method has better performance than several approaches on image retrieval and classification tasks.

\section{REFERENCES}

[1] M. S. Charikar, "Similarity estimation techniques from rounding algorithms," in ACM symposium on Theory of computing, 2002, pp. 380-388.

[2] B. Kulis and K. Grauman, "Kernelized locality-sensitive hashing for scalable image search," in IEEE Conference on Computer Vision and Pattern Recognition, 2009, pp. 2130-2137.

[3] J. Philbin, O. Chum, M. Isard, J. Sivic, and A. Zisserman, "Object retrieval with large vocabularies and fast spatial matching," in IEEE Conference on Computer Vision and Pattern Recognition, 2007, pp. 18.

[4] A. C. Berg, T. L. Berg, and J. Malik, "Shape matching and object recognition using low distortion correspondences," in IEEE Conference on Computer Vision and Pattern Recognition, vol. 1, 2005, pp. 26-33.

[5] J. Hays and A. A. Efros, "Scene completion using millions of photographs," Transactions on Graphics, vol. 26, no. 3, p. 4, 2007.

[6] R. Arandjelović and A. Zisserman, "Three things everyone should know to improve object retrieval," in IEEE Conference on Computer Vision and Pattern Recognition, 2012, pp. 2911-2918.

[7] A. Torralba, R. Fergus, and Y. Weiss, "Small codes and large image databases for recognition," in IEEE Conference on Computer Vision and Pattern Recognition, 2008, pp. 1-8.
[8] J. Wang, S. Kumar, and S.-F. Chang, "Semi-supervised hashing for large-scale search," IEEE Transactions on Pattern Analysis and Machine Intelligence, vol. 34, no. 12, pp. 2393-2406, 2012.

[9] M. Datar, N. Immorlica, P. Indyk, and V. S. Mirrokni, "Locality-sensitive hashing scheme based on p-stable distributions," in Annual Symposium on Computational Geometry, 2004, pp. 253-262.

[10] P. Jain, B. Kulis, and K. Grauman, "Fast image search for learned metrics," in IEEE Conference on Computer Vision and Pattern Recognition, 2008, pp. 1-8.

[11] A. Andoni and P. Indyk, "Near-optimal hashing algorithms for approximate nearest neighbor in high dimensions," in IEEE Symposium on Foundations of Computer Science, 2006, pp. 459-468.

[12] M. Raginsky and S. Lazebnik, "Locality-sensitive binary codes from shift-invariant kernels," Advances in Neural Information Processing Systems, vol. 22, pp. 1509-1517, 2009.

[13] F. J. Huang, Y.-L. Boureau, Y. LeCun et al., "Unsupervised learning of invariant feature hierarchies with applications to object recognition," in IEEE Conference on Computer Vision and Pattern Recognition, 2007, pp. $1-8$.

[14] Y. Gong, S. Kumar, V. Verma, and S. Lazebnik, "Angular quantizationbased binary codes for fast similarity search," in Advances in Neural Information Processing Systems, 2012, pp. 1196-1204.

[15] K. He, F. Wen, and J. Sun, "K-means hashing: An affinity-preserving quantization method for learning binary compact codes," in IEEE Conference on Computer Vision and Pattern Recognition, 2013, pp 2938-2945.

[16] Y. Weiss, A. Torralba, and R. Fergus, "Spectral hashing," in Advances in Neural Information Processing Systems, 2009, pp. 1753-1760.

[17] M. Belkin and P. Niyogi, "Laplacian eigenmaps and spectral techniques for embedding and clustering," Advances in Neural Information Processing Systems, pp. 585-591, 2002.

[18] W. Liu, J. Wang, S. Kumar, and S.-F. Chang, "Hashing with graphs," in International Conference on Machine Learning, 2011, pp. 1-8.

[19] Y. Bengio, O. Delalleau, N. Le Roux, J.-F. Paiement, P. Vincent, and M. Ouimet, "Learning eigenfunctions links spectral embedding and kernel PCA," Neural Computation, vol. 16, no. 10, pp. 2197-2219, 2004.

[20] Y. Gong and S. Lazebnik, "Iterative quantization: A procrustean approach to learning binary codes," in IEEE Conference on Computer Vision and Pattern Recognition. IEEE, 2011, pp. 817-824.

[21] C. Strecha, A. M. Bronstein, M. M. Bronstein, and P. Fua, "LDAHash: Improved matching with smaller descriptors," IEEE Transactions on Pattern Analysis and Machine Intelligence, vol. 34, no. 1, pp. 66-78, 2012.

[22] R. Salakhutdinov and G. Hinton, "Semantic hashing," International Journal of Approximate Reasoning, vol. 50, no. 7, pp. 969-978, 2009.

[23] M. Norouzi and D. M. Blei, "Minimal loss hashing for compact binary codes," in International Conference on Machine Learning, 2011, pp. 353-360.

[24] J. Wang, S. Kumar, and S.-F. Chang, "Sequential projection learning for hashing with compact codes," in International Conference on Machine Learning, 2010, pp. 1127-1134.

[25] Y. Mu, J. Shen, and S. Yan, "Weakly-supervised hashing in kernel space," in IEEE Conference on Computer Vision and Pattern Recognition, 2010, pp. 3344-3351.

[26] W. Liu, J. Wang, R. Ji, Y.-G. Jiang, and S.-F. Chang, "Supervised hashing with kernels," in IEEE Conference on Computer Vision and Pattern Recognition, 2012, pp. 2074-2081.

[27] B. Kulis, P. Jain, and K. Grauman, "Fast similarity search for learned metrics," IEEE Transactions on Pattern Analysis and Machine Intelligence, vol. 31, no. 12, pp. 2143-2157, 2009.

[28] F. Shen, C. Shen, W. Liu, and H. T. Shen, "Supervised discrete hashing," IEEE Conference on Computer Vision and Pattern Recognition, pp. 3745, 2015.

[29] J. Deng, W. Dong, R. Socher, L.-J. Li, K. Li, and L. Fei-Fei, "Imagenet: A large-scale hierarchical image database," in IEEE Conference on Computer Vision and Pattern Recognition, 2009, pp. 248-255.

[30] N. Verma, D. Mahajan, S. Sellamanickam, and V. Nair, "Learning hierarchical similarity metrics," in IEEE Conference on Computer Vision and Pattern Recognition, 2012, pp. 2280-2287.

[31] B. Zhao and E. P. Xing, "Hierarchical feature hashing for fast dimensionality reduction," in IEEE Conference on Computer Vision and Pattern Recognition, 2014, pp. 2051-2058. 
[32] B. Kulis and T. Darrell, "Learning to hash with binary reconstructive embeddings," in Proceedings of the Neural Information Processing Systems Conference, 2009, pp. 1042-1050.

[33] A. Torralba, R. Fergus, and W. T. Freeman, "80 million tiny images: A large data set for nonparametric object and scene recognition," IEEE Transactions on Pattern Analysis and Machine Intelligence, vol. 30, no. 11, pp. 1958-1970, 2008.

[34] A. Krizhevsky, I. Sutskever, and G. E. Hinton, "Imagenet classification with deep convolutional neural networks," in Proceedings of the Neural Information Processing Systems Conference, 2012, pp. 1097-1105.

[35] V. Erin Liong, J. Lu, G. Wang, P. Moulin, and J. Zhou, "Deep hashing for compact binary codes learning," in IEEE Conference on Computer Vision and Pattern Recognition, 2015, pp. 2475-2483.

[36] J. Deng, A. C. Berg, and L. Fei-Fei, "Hierarchical semantic indexing for large scale image retrieval," in IEEE Conference on Computer Vision and Pattern Recognition. IEEE, 2011, pp. 785-792.

[37] Y. Gong, M. Pawlowski, F. Yang, L. Brandy, L. Boundev, and R. Fergus, "Web scale photo hash clustering on a single machine," in IEEE Conference on Computer Vision and Pattern Recognition, 2015, pp. 1927.

[38] Y. Nesterov, Introductory lectures on convex optimization. Springer Science \& Business Media, 2004, vol. 87.

[39] D. G. Lowe, "Distinctive image features from scale-invariant keypoints," International Journal of Computer Vision, vol. 60, no. 2, pp. 91-110, 2004.

[40] C. H. Lampert, H. Nickisch, and S. Harmeling, "Learning to detect unseen object classes by between-class attribute transfer," in IEEE Conference on Computer Vision and Pattern Recognition, 2009, pp. 951958

[41] Y. Jia, "Caffe: An open source convolutional architecture for fast feature embedding," 2013.

[42] K. Crammer and Y. Singer, "On the algorithmic implementation of multiclass kernel-based vector machines," The Journal of Machine Learning Research, vol. 2, pp. 265-292, 2002.

[43] O. Dekel, J. Keshet, and Y. Singer, "Large margin hierarchical classification," in International Conference on Machine Learning, 2004, p. 27.

[44] D. Koller and M. Sahami, "Hierarchically classifying documents using very few words," 1997. 